\title{
Evaluation of the statutory surveillance system for invasive MRSA infections in Germany, 2016-2017
}

\author{
Viktoria Schönfeld ${ }^{1,2,3^{*}}$ (D, M. Diercke ${ }^{1}$, A. Gilsdorf ${ }^{1}$, T. Eckmanns $^{1}$ and J. Walter ${ }^{1}$
}

\begin{abstract}
Background: Mandatory notification of invasive methicillin-resistant Staphylococcus aureus (MRSA) infections was introduced for laboratories in Germany in 2009. The aims were to support local health authorities (LHAs) in their mandate to prevent and control infections in hospitals and to improve population-based nationwide surveillance of healthcare associated infections. We evaluated the MRSA surveillance system to assess whether its aims were met and to identify areas for improvement.

Methods: Using the updated guidelines for evaluating public health surveillance systems by the Centers for Disease Control and Prevention we assessed the attributes simplicity, timeliness, data quality, acceptability, and usefulness. In 2016/2017 we interviewed staff in LHAs, state health authorities (SHAs), and laboratories and analyzed surveillance data of cases notified between 2009 and 2016.

Results: We interviewed 10\% of LHAs $(n=38), 63 \%$ of SHAs $(n=10), 5$ selected laboratories and analyzed information on 27,706 notified MRSA cases. LHAs reported that on receiving notifications from laboratories they contacted hospitals for clinical information, which was time-consuming and complicated as physicians were hard to reach or refused to answer questions, citing doctor-patient confidentiality. LHAs suggested reducing the amount of information collected as some clinical information was unnecessary for implementing control measures. LHAs stated that they received notifications on time, however surveillance data analysis showed some delay. Data completeness exceeded $90 \%$ for most variables, however it was only $68 \%$ and $80 \%$ for dates of disease onset and hospital admission respectively making it impossible to discriminate between hospital and community acquired infections in half of the cases. The surveillance system was well accepted by half of the interviewees. A third however stated that the benefits of the surveillance system were outweighed by the work associated with it. The majority rated the system to be useful for recognizing trends in the MRSA incidence and the ability to check up on infection control measures in hospitals.
\end{abstract}

Conclusions: The surveillance system proved to be useful by fulfilling its aims. It was timely, acceptable and provided complete data for most variables. However, the system was complicated; ensuring that only relevant variables are reported could simplify the system without losing any of its usefulness.

Keywords: Disease notification, Program evaluation, Methicillin-resistant Staphylococcus aureus, Telephone interviews

\footnotetext{
* Correspondence: SchoenfeldV@rki.de

'Department for Infectious Disease Epidemiology, Robert Koch Institute,

Berlin, Germany

${ }^{2}$ Postgraduate Training for Applied Epidemiology, Robert Koch Institute,

Berlin, Germany

Full list of author information is available at the end of the article
}

(c) The Author(s). 2018 Open Access This article is distributed under the terms of the Creative Commons Attribution 4.0 International License (http://creativecommons.org/licenses/by/4.0/), which permits unrestricted use, distribution, and reproduction in any medium, provided you give appropriate credit to the original author(s) and the source, provide a link to the Creative Commons license, and indicate if changes were made. The Creative Commons Public Domain Dedication waiver (http://creativecommons.org/publicdomain/zero/1.0/) applies to the data made available in this article, unless otherwise stated. 


\section{Background}

Methicillin-resistant Staphylococcus aureus (MRSA) is the multidrug-resistant pathogen that most frequently causes hospital acquired infections in Germany $[1,2]$. One mechanism to control communicable diseases is the implementation of effective surveillance systems. Surveillance enables the identification of temporal and regional trends, the evaluation of the effects of interventions, and thereby allows the implementation of effective infection prevention and control (IPC) measures and raises awareness of public health problems [3].

Germany introduced mandatory notification for invasive MRSA infections for laboratories in 2009. The new MRSA surveillance was integrated into the statutory notifiable diseases surveillance system. The aims of the MRSA surveillance are to improve nationwide surveillance of healthcare associated infections by introducing population-based MRSA surveillance and to strengthen local health authorities (LHAs) in their capacity to effectively prevent and control healthcare associated infections [4]. However, it has been questioned whether or not this surveillance system actually contributes to the prevention of nosocomial infections [5]. In addition, periodical evaluations of surveillance systems are crucial in order to ensure that systems fulfill their aims and to implement improvements as required [6-8]. The German surveillance system for MRSA has not yet been evaluated in detail.

We evaluated the surveillance system for invasive MRSA infections by analyzing surveillance data and by interviewing key users of the system including staff in
LHAs, state health authorities (SHAs), and laboratories to assess whether aims of the system are being met and to identify areas for potential improvement.

\section{Methods \\ Evaluation}

We used methods described in the updated guidelines for evaluating public health surveillance systems by the Centers for Disease Control and Prevention (CDC), which we modified to fit the surveillance system under evaluation [8] (Table 1). We engaged key stakeholders (at SHAs, LHAs, and the national public health institute, the Robert Koch-Institute (RKI)) in the development of the study design and of a questionnaire. We assessed the attributes simplicity, data quality, timeliness, acceptability, and usefulness. The evaluation was divided into two parts. First, we interviewed staff from LHAs, SHAs, and laboratories using a semi-structured questionnaire, which contained open and closed questions. Second, we analyzed surveillance data of cases notified between 2009 and 2016.

\section{Selection of participants and interviews}

We recruited SHAs by asking them at a meeting whether they would participate in the study and sent out information afterwards. In order to recruit LHAs, we asked SHAs to forward an invitation and information sheet about the study to the LHAs in their federal states. We planned to interview 2 to 5 LHAs per federal state depending on the population size. If more than the planned number of LHAs per state were interested in participating in the study, we selected LHAs by including a balanced number

Table 1 Methods and measures used for assessing attributes of statutory MRSA surveillance, Germany 2016/2017 (based on CDC guidelines)

\begin{tabular}{|c|c|c|}
\hline Attribute & Methods & Measures and questions used to assess the attribute \\
\hline Simplicity & Interviews & $\begin{array}{l}\text { - Description of the surveillance system } \\
\text { - Amount and type of data on cases } \\
\text { (for case definition and other data) } \\
\text { - Method of data collection including number } \\
\text { and types of reporting sources } \\
\text { - Time for collecting and managing the data (e.g. data entry) }\end{array}$ \\
\hline Timeliness & Data analysis and interviews & $\begin{array}{l}\text { - Time elapsed between diagnosis and notification } \\
\text { and between notification and data transmission }\end{array}$ \\
\hline Data quality & Data analysis and interviews & $\begin{array}{l}\text { - Completeness of surveillance data } \\
\text { - Presence of data quality checks }\end{array}$ \\
\hline Acceptability & Data analysis and interviews & $\begin{array}{l}\text { - Quantitative measures: reporting rate, data completeness, } \\
\text { completion rate of investigations, timeliness } \\
\text { - Perceived public health relevance of disease } \\
\text { - Dissemination of aggregated data to interested parties } \\
\text { - Assessment of effort in relation to usefulness of the system } \\
\text { - Statutory requirements for reporting and data collection }\end{array}$ \\
\hline Usefulness & Interviews & $\begin{array}{l}\text { - Does the system contribute to a timely implementation } \\
\text { of prevention and control measures? } \\
\text { - Does the system provide estimates for morbidity? } \\
\text { - Does the system detect trends? } \\
\text { - Does the system lead to improved clinical practices? } \\
\text { - Assessment of usefulness on } 1 \text { to } 10 \text { scale }\end{array}$ \\
\hline
\end{tabular}


of LHAs in urban and rural districts, with small and large population sizes, and with high and low incidence of notified invasive MRSA infections. In addition, LHAs were asked to provide names of notifying laboratories in their district during the interviews and a convenience sample of five laboratories from differently sized districts was scheduled for interviews.

The semi-structured phone interviews with representatives of LHAs, SHAs and laboratories were conducted by two researchers where possible. Interviewees were asked for consent to be re-contacted in case a clarification of their responses was required.

\section{Data analysis}

Data analysis was conducted for all electronically transmitted cases of invasive MRSA infections notified to the Robert Koch Institute between 2009 and 2016 which met the surveillance case definition. Data were extracted from the electronic surveillance database SurvNet@RKI [9] on March 2nd, 2017 and subsequently analyzed using Excel (Microsoft Excel 2010) and Stata (StataSE 14). Data was considered to be complete if "yes", "no", or a date was specified for a variable. If LHAs had indicated that a result was either not investigated or could not be ascertained, data was considered to be incomplete.

\section{Confidentiality and ethical statement}

Participation in the interviews was voluntary and interviewees' personal data (name of interviewee, name of LHA, federal state, etc.) were pseudonymized. Surveillance data contained only variables as required by the German Infection Protection Act. Only aggregated data are published in the results.

\section{Results}

Between June 2016 and January 2017, we interviewed representatives of 38 LHAs (10\% of all LHAs), 10 SHAs (63\% of all SHAs), and 5 laboratories. Furthermore, we analyzed data of 27,706 cases of invasive MRSA infection notified between 2009 and 2016 .

\section{Description of the system}

The surveillance system for invasive MRSA infections is integrated into the statutory notification system regulated by the German Infection Protection Act. It is a comprehensive, passive, case-based surveillance system requiring laboratories to notify MRSA detected in blood or cerebrospinal fluid (CSF). The case definition includes laboratory-confirmed cases irrespective of the clinical presentation [10]. Laboratory confirmation requires the culture of S. aureus from blood or CSF and the detection of methicillin resistance either by susceptibility testing or detection of the mecA gene [11].
LHAs should be notified by laboratories about a case of invasive MRSA infection within $24 \mathrm{~h}$. Notifications are usually paper-based and must include but are not limited to demographic information on the patient as well as type and result of the diagnostic tests. LHAs then enter the data into an electronic database and transmit pseudonymized data supplemented by information on clinical presentation and outcome, date of disease onset, and mode of transmission electronically to their SHA within one workday. If available, additional information on the focus of the infection such as invasive devices and catheters, trauma, or other localizations of MRSA infections should also be collected and transmitted. The data is then transmitted electronically by the SHA to the RKI within the next workday (Fig. 1) [12].

According to the Infection Protection Act, laboratories are obliged to make the notification to the LHA responsible for the district from where the sample was sent to the laboratory, which is usually a hospital. The Infection Protection Act also states that the LHA responsible for the district of the patient's residence is in charge of transmitting the data to their SHA. If the patient's residence and the hospital are in two different districts, the LHA receiving the notification and the LHA in charge of transmitting the data to the SHA are not the same. In this situation the first LHA (hospital-LHA) has to forward the notification to the second LHA (patient-LHA). There is no legal regulation as to which LHA has to investigate clinical presentation and focus of infection with the hospital.

\section{Simplicity}

All laboratories reported the notification process to be simple and quick as it had been largely automatized. Notification forms were mostly faxed or, by one laboratory,

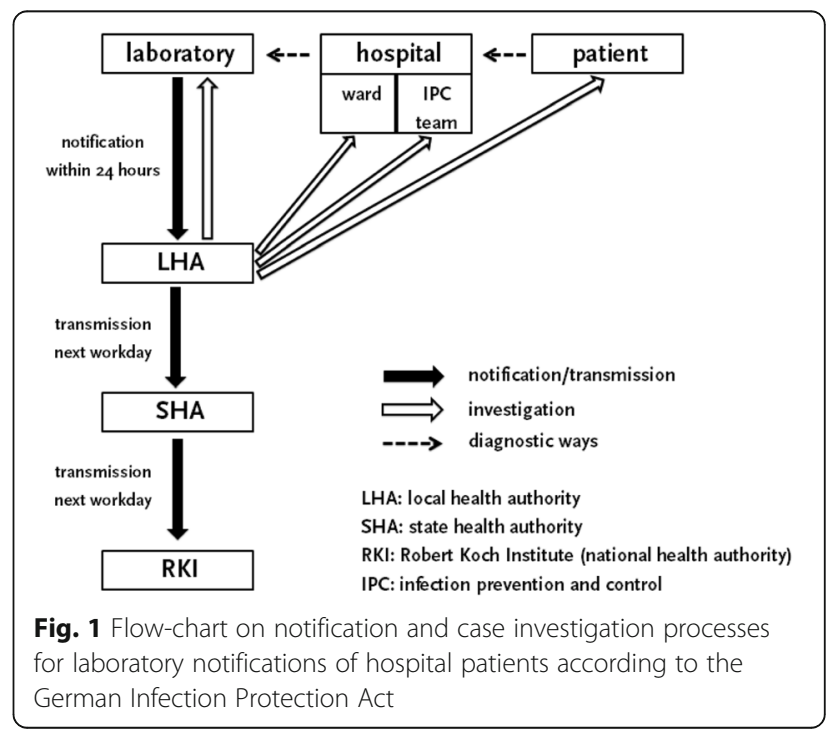


sent by mail to the LHA. Once the notification form had arrived at the LHA, data was entered into the electronic database. Subsequently either health inspectors $(73 \% ; 27$ of 37 LHAs), physicians (16\%; 6 of 37 LHAs), or other staff members (11\%; 4 of 37 LHAs) collected information that had not been available in the laboratory notification form. This included variables such as clinical presentation or the focus of the MRSA infection. They also checked whether prevention and control measures had been implemented in the hospital. Specific data collection forms for the investigation of invasive MRSA infections were used by $84 \%$ of LHAs (32 of 38). These were either sent to the hospital or used during a telephone interview. LHAs investigated either by email, mail, or fax $(51 \% ; 19$ of 37$)$ or by phone $(49 \% ; 18$ of 37$)$. People contacted for this purpose included the treating physician (92\%; 34 of 37), the IPC team of the hospital (76\%; 28 of 37), and the patients and their families (16\%; 6 of 37) (multiple answers were possible). About a third of LHAs (36\%; 13 of 36) reported that some hospitals in their districts sent all the required information on their own initiative after they had been alerted by the laboratory about a case of an invasive MRSA infection without requiring any further queries from the LHAs.

Applying the case definition was perceived as simple by most of the interviewees in LHAs; some reported that there had been some difficulties during the initial phase of the surveillance. Two LHAs stated that health inspectors found it difficult to interpret laboratory reports and that in their opinion physicians should conduct the investigations with laboratories and hospitals. Three LHAs reported that the clinical presentation or the cause of death was not always clear. About half of the LHAs reported investigations to take longer than $30 \mathrm{~min}$ and data collection longer than 3 days to complete (Fig. 2). They reported the following reasons for delays in completing the investigations: physicians in the hospitals were hard to reach, especially if hospitals in other districts were involved; physicians sometimes did not answer the questions due to time constraints or due to misled doctor-patient confidentiality concerns; or patients had already been discharged at the time of the case investigation often due to delayed notifications by the laboratory. Several LHAs suggested omitting clinical variables relating to symptoms or the focus of the infection as these were often laborious and time-consuming to collect.

When patients were hospitalized in another district than their place of residence, 32\% (12 of 38) of the hospital-LHAs (LHA of the district where the hospital is located) conducted the case investigations in the hospitals themselves, $5 \%$ ( 2 of 38 ) decided on a case-by-case basis which LHA had to investigate, and 63\% (24 of 38) forwarded the notification forms to the patient-LHA (LHA of the district of the patient's residence) without their own investigations.

\section{Timeliness}

\section{Diagnosis to notification}

All laboratories reported that they sent out notifications on the day the diagnosis was confirmed. However, one laboratory sent notifications forms by regular mail, which can take longer than 1 day. 86\% (32 of 37) of LHAs reported that they received the notifications on the day of or the day following diagnosis. Analysis of surveillance data showed that median duration from diagnosis to the receipt of the notification at LHAs was 2 days (Table 2). Although delayed notifications were not specifically identified by LHAs as an issue in terms of implementation of control measures, some did report that this could mean that data collection in hospitals was difficult or impossible.

\section{Notification to data entry and data entry to arrival at RKI}

Median duration from the receipt of a notification to its entry in the electronic system at the LHAs was less than 1 day and from data entry to arrival of data at RKI 1 day (Table 2). Data transmission from LHAs to SHAs within $24 \mathrm{~h}$ was reported by $60 \%$ of SHAs (6 of 10). Three SHAs reported that a few LHAs did not transmit data daily when there were staff shortages, when LHAs wanted the data to be complete before entering the case into the database, or when two LHAs were involved in investigations and forwarding of notification forms between LHAs was prolonged.

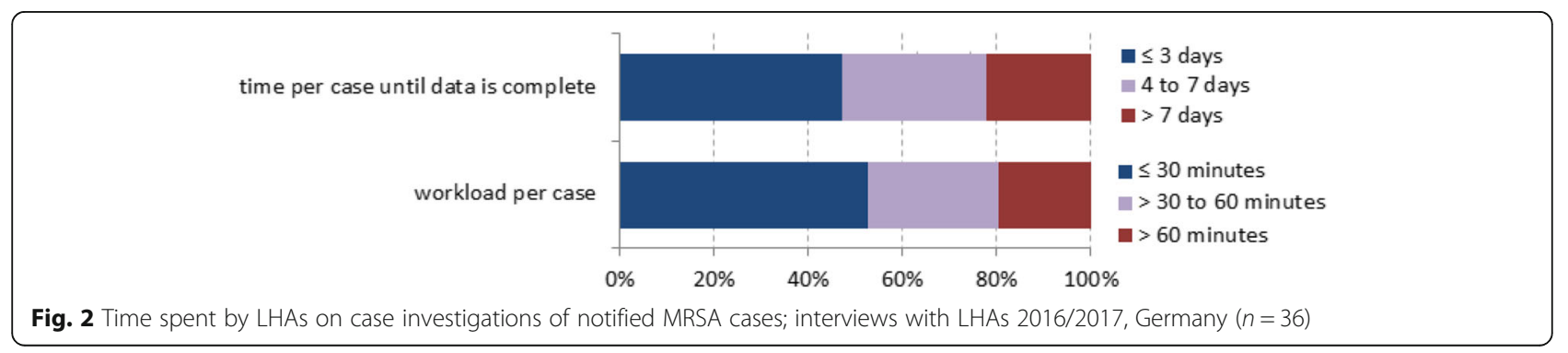


Table 2 Timeliness of notification and data transmission of notified MRSA cases; Germany 2009-2016

\begin{tabular}{lll}
\hline & Median [days] & $I^{\mathrm{a}}[\mathrm{days}]$ \\
\hline Diagnosis to notification $(n=1530)$ & 2 & $1 ; 4$ \\
Notification to data entry at LHA $(n=2245)$ & 0 & $0 ; 0$ \\
Data entry at LHA to data arrival at RKI $(n=2245)$ & 1 & $1 ; 2$ \\
\hline
\end{tabular}

a IQR: interquartile range

\section{Data quality}

\section{Data completeness}

Data completeness of the 27,706 cases notified between 2009 and 2016 reached $96 \%$ to $100 \%$ for variables related to demographic characteristics and clinical presentation. Data completeness for diagnostic methods was $95 \%$ for the "sample type" for the whole period and improved markedly for "type of susceptibility testing" from $11 \%$ in 2009 to $95 \%$ in 2016. Apart from date of death, date variables were often not specified: the date of disease onset and the date of hospital admission had been specified for $68 \%$ and $80 \%$ of the cases respectively; and for only $56 \%$ both date variables had been specified. Completeness of additional information like symptoms and risk factors could not be assessed as a checkbox option "none" was not provided for these variables.

\section{Data quality management}

Quality checks were performed by $97 \%$ of LHAs (36 of 37 ) on the data entered into the database. All LHAs (36 of 36) tried to identify and delete duplicate notifications by either automatic or manual procedures. Additional quality checks were reported to be conducted by $72 \%$ of LHAs (26 of 36); most of them specified that the responsible SHAs checked for missing or implausible data. The majority of SHAs (70\%; 7 of 10) reported that they performed these quality checks on every single case.

\section{Acceptability}

According to an analysis of the surveillance data, since 2009 all but one LHA had transmitted at least one case of invasive MRSA infection. Case investigations could not always be completed by $15 \%$ of LHAs ( 5 of 33). This was partially due to the fact that doctors felt that they would breach doctor-patient confidentiality if they provided the required information. Delayed notifications made it also more difficult to collect all the data as patient charts were no longer easily accessible. Some interviewees commented that the workload associated with the investigation of invasive MRSA infections was justified given the severity of the disease. Aggregated data was disseminated to interested parties by LHAs, but one laboratory stated that it would be useful to get feedback on the conclusions drawn from the data. Most LHAs $(86 \% ; 32$ of 37$)$ reported that they were able to collaborate well with hospitals. The majority of SHAs and LHAs stated that the benefits of the surveillance system were worth the workload associated with investigating cases of invasive MRSA infections (Fig. 3).

\section{Usefulness}

According to LHAs and SHAs the usefulness of the system was partly due to the epidemiological data it delivered so that trends could be identified. Some respondents stated that notified invasive MRSA infections were a good proxy indicator for trends in all MRSA infections or even colonizations, conversely others voiced the opinion that the system only detected the "tip of the iceberg" and that the true burden could not be estimated using notified cases. Almost all interviewees (76\% of LHAs and $90 \%$ of SHAs) analyzed their data, although some LHAs stated that they had too few cases to conduct any further analysis for MRSA only. Most interviewed sites (90\%; 43 of 48) made use of published surveillance data, $48 \%$ using data from their federal state and 54\% using national data published by RKI (multiple answers were possible). They either used the data for routine reports,

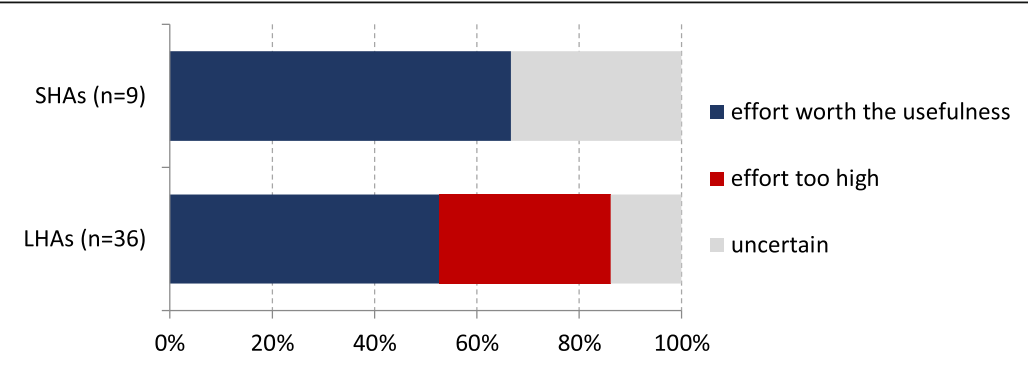

Fig. 3 Balance of effort and usefulness of the surveillance system for invasive MRSA infections in Germany; interviews with SHAs and LHAs 2016/2017 $(n=45)$ 
for queries from press or policy makers, or to inform local networks for control of antimicrobial resistance.

Another benefit reported by LHAs was that LHAs took action following notifications and provided advice regarding control measures where required. However, some LHAs also reported that additional advice from their side was usually not required as they already knew which hospitals had hygiene guidelines and whether they were being followed. Some LHAs performed inspections of hospital wards when a case had been notified but most of them only did this in response to clusters of MRSA cases which rarely were invasive infections (Fig. 4). Two LHAs reported that they had detected hygiene deficiencies due to notified invasive MRSA infections. LHAs used case investigations following notifications to assess whether hospitals were complying with hygiene guidelines. This was reflected in the data collection forms, most of which (84\%) contained questions regarding mode of transmission or prevention and control measures. Several LHAs reported that case investigations led to an intensified contact to hospitals, a better collaboration in terms of hygiene, and a better acceptance of prevention measures from the hospitals.

On a scale of 1 to 10 for the usefulness of the system, LHAs allocated a median of 7 points (range 2 to 10) and SHAs 6.75 points (range 2 to 10) to the surveillance system.

\section{Discussion}

We conducted the first detailed evaluation of the German mandatory surveillance system for invasive MRSA infections including interviews with stakeholders and an analysis of surveillance data. We found that overall the aims of the system were met. However, by assessing the individual attributes of surveillance systems as recommended by the guidelines of the CDC [8], we also identified some areas for improvement (Table 3).

The MRSA surveillance aims to improve surveillance of healthcare associated infections and provides nationwide and mostly complete data on the epidemiology of invasive MRSA infections. This information is used to inform members of local networks for control of antimicrobial resistance, policy makers, and the public. The second aim, which is to support LHAs in preventing and controlling healthcare associated infections, is met by providing an additional opportunity for LHAs to check on measures undertaken in hospitals. MRSA surveillance had also led to a closer collaboration between LHAs and hospitals and might contribute towards better compliance with guidelines on infection prevention and control in hospitals.

However, when LHAs conduct case investigations in hospitals in other districts (because their resident is hospitalized in a different district), they cannot check on infection control measures in these hospitals as they have no regulatory supervision over these hospitals (according to the Infection Protection Act). In order to avoid this problem we recommend that LHAs of the district of the hospitals investigate and check on control measures if necessary and then forward the information to the patient-LHA for data transmission to the SHA. The results of this evaluation will inform the development of a new electronic reporting system with a particular focus on improving the exchange of data and the collaboration between LHAs.

Though the system was assessed to be useful, we found it to be complicated, resulting in a considerable workload for data collection by LHAs. Collecting data in hospitals was perceived to be labor intensive and time-consuming by many LHAs with almost half of the interviewed LHAs requiring more than $30 \mathrm{~min}$ for each case investigation. When the system was introduced, an average 26 min were estimated to be necessary for managing a case and 2000 cases were expected annually [4]. As currently about 3000 cases are notified annually, the resulting workload is considerably higher than estimated. Reducing the amount of data to be collected per case would address this. Several LHAs suggested that information on symptoms and focus of infection was unnecessary. They felt this information was difficult to ascertain and that it was not used by the LHAs. Omitting these variables might help making the system simpler and less resource-intensive and improve acceptability without reducing its usefulness.

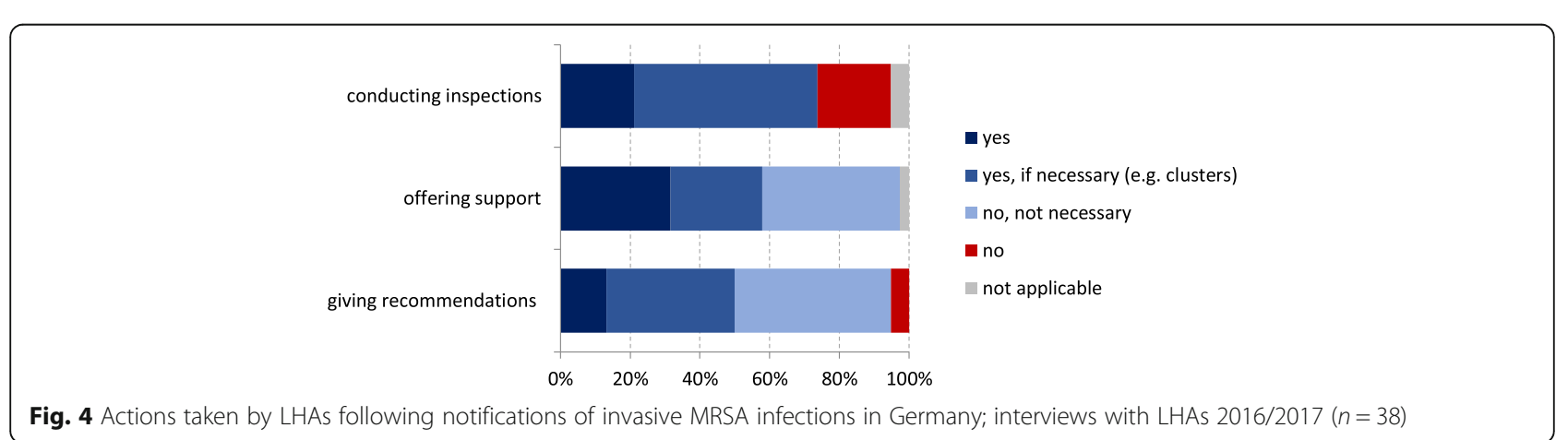

Fig. 4 Actions taken by LHAs following notifications of invasive MRSA infections in Germany; interviews with LHAs 2016/2017 ( $n=38)$ 
Table 3 Recommendations for improving statutory MRSA surveillance; Germany 2016/2017

\begin{tabular}{|c|c|}
\hline Attribute assessed & Recommendations for improvement \\
\hline Simplicity & $\begin{array}{l}\text { - Reducing variables on clinical symptoms } \\
\text { - Use data collection forms } \\
\text { - Deposit data collection forms with IPC team } \\
\text { of hospital } \\
\text { - Clarify that hospitals are obliged legally to } \\
\text { collaborate with LHAs on data collection }\end{array}$ \\
\hline Data quality & $\begin{array}{l}\text { - Clarify that hospitals are obliged legally to } \\
\text { collaborate with LHAs on data collection } \\
\text { - Include variables to be able to discriminate } \\
\text { between hospital and community acquired } \\
\text { infections }\end{array}$ \\
\hline Timeliness & - None \\
\hline Acceptability & - Reducing variables on clinical symptoms \\
\hline Usefulness & $\begin{array}{l}\text { - LHA with regulatory supervision should } \\
\text { investigate and check on IPC measures in } \\
\text { hospital } \\
\text { - Include variables to be able to discriminate } \\
\text { between hospital and community acquired } \\
\text { infections }\end{array}$ \\
\hline
\end{tabular}

Another approach to simplify data collection would be to provide the IPC team of the hospital with data collection forms which they could complete independently and submit to the LHAs. However, some LHAs considered speaking directly to the affected wards useful. LHAs will need to individually find a balance between the simplest way of data collection and the need for dialogue with the hospital.

A significant barrier to data collection was the refusal by some physicians to share detailed information due to concerns around patients' data confidentially. In a response to this problem, the German law has recently been changed $[13,14]$. Hospitals are now explicitly obliged to collaborate with LHAs on the collection of surveillance data. This will facilitate data collection for LHAs in the future.

Another issue that we identified was the inability to discriminate between hospital and community acquired MRSA infections for many of the notifications. This is due to low levels of data completeness for the dates of disease onset and of hospital admission, which are often used to identify hospital acquired infections. From the current surveillance system, it is also impossible to determine whether patients were transferred from other institutions where transmission might also have occurred, prior to hospitalization. We suggest this should be captured by the surveillance system, as it is for example the case in the English or US American system [15, 16].

This study had some limitations due to a possible selection bias. The selection of the LHAs was not random, but based on voluntary participation. However, $10 \%$ of LHAs were included in the study and their selection was purposefully diverse with the inclusion of small and large
LHAs in rural and urban areas in Germany with low and high MRSA incidence. Due to voluntary participation, the results may not represent the view of less interested LHAs that might have more or less problems with the surveillance system than the interviewed sites. However, analysis of interviews with LHAs as well as SHAs showed that there were no more new aspects introduced during later rounds of interviews suggesting that data saturation had been reached. In addition, we presented the results to LHAs and SHAs at a conference and at meetings asking for their comments, which were generally consenting.

\section{Conclusions}

In summary we found the surveillance system for invasive MRSA infections to be useful, timely, and well accepted with mostly good data quality and to be fulfilling its aims. Reducing the amount of information to be collected by LHAs could make the system simpler and less resourceintensive without impacting on its usefulness and may even increase its acceptance especially in LHAs.

\section{Abbreviations \\ CDC: Centers for Disease Control and Prevention; CSF: Cerebrospinal fluid; IPC: Infection prevention and control; IQR: Interquartile range; LHA: Local health authority; MRSA: Methicillin-resistant Staphylococcus aureus; RKI: Robert} Koch Institute; SHA: State health authority

\section{Acknowledgements}

The authors thank all the participants from local and state health authorities and laboratories. We thank Doris Altmann and Dr. Hermann Claus for contributing to the analysis regarding timeliness of the system. We also thank Dr. Katharina Alpers from the PAE program for reviewing and Janine Thoulass for proofreading the manuscript.

\section{Availability of data and materials}

The original datasets regarding the interviews used and/or analyzed during the current study are available from the corresponding author on reasonable request. Aggregated surveillance data for notifiable diseases is available from https://survstat.rki.de/.

\section{Authors' contributions}

VS, MD, AG, TE, and JW contributed to the design of this study. Interviews were conducted by VS, MD, and JW. Analyses were performed by VS, and VS, $M D, A G, T E$, and JW contributed to the interpretation of data and elaborated the recommendations. All authors contributed to writing the manuscript and approved the final version.

\section{Ethics approval and consent to participate}

For analysis, data from interviews was pseudonymized and anonymized surveillance data was used. Only aggregated data is published in the article. Participation in the study was voluntary and all participants were informed about the objectives of the study. As the study did not contain any personal data and interviews did not contain sensitive questions, no ethics approval was considered necessary according to the Professional Code for Physicians in Germany (https://www.bundesaerztekammer.de/fileadmin/user_upload/ downloads/pdf-Ordner/MBO/MBO-AE_EN_2018.pdf).

Consent for publication

Not applicable.

Competing interests

The authors declare that they have no competing interests. 


\section{Publisher's Note}

Springer Nature remains neutral with regard to jurisdictional claims in published maps and institutional affiliations.

\section{Author details}

${ }^{1}$ Department for Infectious Disease Epidemiology, Robert Koch Institute, Berlin, Germany. ${ }^{2}$ Postgraduate Training for Applied Epidemiology, Robert Koch Institute, Berlin, Germany. ${ }^{3}$ European Programme for Intervention Epidemiology Training, ECDC, Stockholm, Sweden.

Received: 19 December 2017 Accepted: 15 August 2018

Published online: 24 August 2018

\section{References}

1. Geffers C, Gastmeier P. Nosocomial infections and multidrug-resistant organisms in Germany: epidemiological data from KISS (the hospital infection surveillance system). Dtsch Arztebl Int. 2011;108(6):87-93.

2. Gastmeier P, Geffers C, Herrmann M, Lemmen S, Salzberger B, Seifert H, et al. Nosokomiale Infektionen und Infektionen mit multiresistenten Erregern Häufigkeit und Sterblichkeit. [Nosocomial infections and infections with multidrug-resistant pathogens - frequency and mortality]. Dtsch Med Wochenschr. 2016;141(06):421-6

3. Buehler JW. Surveillance. In: Rothmann KJ, Greenland S, Lash TL, editors. Modern Epidemiology. Philadelphia: Lippincott Williams \& Wilkins; 2008. p. 459-80

4. Federal Council of Germany. Verordnung zur Anpassung der Meldepflicht nach $\S 7$ des Infektionsschutzgesetzes an die epidemische Lage (Labormeldepflicht-Anpassungsverordnung - LabMeldAnpV) [Regulation for adjustment of statutory notifications to the epidemiological situation according to $\S 7$ Infection Protection Act]. Berlin: Federal Council of Germany; 2009.

5. Heudorf U, Mischler D, Bobyk D, Bornhofen B, Maiwald M, Merbs R, et al. Meldepflicht MRSA in Blutkulturen - Daten und Erfahrungen des MRE-Netz Rhein-Main, 2011. [MRSA bloodstream infections according to the German obligation for notification - data and experience of the MDRO Network Rhine-Main, Germany, 2011]. Gesundheitswesen. 2014;76(6):385-91.

6. Drewe JA, Hoinville LJ, Cook AJ, Floyd T, Stark KD. Evaluation of animal and public health surveillance systems: a systematic review. Epidemiol Infect. 2012;140(4):575-90.

7. Krause G. From evaluation to continuous quality assurance of surveillance systems. Eur Secur. 2006;11(11):204-5.

8. German RR, Lee LM, Horan JM, Milstein RL, Pertowski CA, Waller MN. Updated guidelines for evaluating public health surveillance systems: recommendations from the guidelines working group. MMWR Recomm Rep. 2001;50(Rr-13):1-35.

9. Faensen D, Claus H, Benzler J, Ammon A, Pfoch T, Breuer T, et al. SurvNet@RKI--a multistate electronic reporting system for communicable diseases. Euro Surveill. 2006:11(4):100-3.

10. Robert Koch Institute. Falldefinitionen des Robert Koch-Instituts zur Übermittlung von Erkrankungs- oder Todesfällen und Nachweisen von Krankheitserregern. [Case definitions of the Robert Koch Institute for the transmission of cases or deaths and the detection of pathogens] 2015. http://www.rki.de/DE/Content/Infekt/IfSG/Falldefinition/Downloads/ Falldefinitionen_des_RKI.pdf?_blob=publicationFile. Accessed 28 Nov 2017

11. Beck WD, Berger-Bachi B, Kayser F. Additional DNA in methicillin-resistant Staphylococcus aureus and molecular cloning of mec-specific DNA. J Bacteriol. 1986;165(2):373-8.

12. Federal Ministry of Justice and Consumer Protection. Infection Protection Act. Bundesgesetzblatt. 2000:Part I (33):1045-71. Updated 2017.

13. German Federal Parliament. Gesetz zur Modernisierung der epidemiologischen Überwachung übertragbarer Krankheiten. In: Law for modernising the surveillance of transmissible diseases; 2017.

14. Diercke M. Änderungen des Infektionsschutzgesetzes, Juli 2017. [changes to the infection protection act, July 2017]. Epid Bull. 2017;31:309-10.

15. Johnson AP, Davies J, Guy R, Abernethy J, Sheridan E, Pearson A, et al. Mandatory surveillance of methicillin-resistant Staphylococcus aureus (MRSA) bacteraemia in England: the first 10 years. J Antimicrob Chemother. 2012;67(4):802-9.

16. Emori TG, Culver DH, Horan TC, Jarvis WR, White JW, Olson DR, et al. National nosocomial infections surveillance system (NNIS): description of surveillance methods. Am J Infect Control. 1991;19(1):19-35.

Ready to submit your research? Choose BMC and benefit from:

- fast, convenient online submission

- thorough peer review by experienced researchers in your field

- rapid publication on acceptance

- support for research data, including large and complex data types

- gold Open Access which fosters wider collaboration and increased citations

- maximum visibility for your research: over $100 \mathrm{M}$ website views per year

At $\mathrm{BMC}$, research is always in progress.

Learn more biomedcentral.com/submissions 